\title{
Localized electron state in a T-shaped confinement potential
}

\author{
L. A. Openov \\ Moscow State Engineering Physics Institute (Technical University), \\ Kashirskoe sh. 31, Moscow 115409, Russia
}

\begin{abstract}
We consider a simple model of an electron moving in a T-shaped confinement potential. This model allows for an analytical solution that explicitly demonstrates the existence of laterally bound electron states in quantum wires obtained by the cleaved edge overgrowth technique.
\end{abstract}


In recent years, semiconductor nanostructures have attracted much attention due to both a great variety of their possible applications in electronic devices and a new interesting physics emerged to describe their peculiar characteristics [1]. Among other research directions, there are intensive studies of the so called quantum wires [2], in which electrons are confined in two spatial dimensions within a nanometersize region, while being allowed to move freely in the third direction (an axis of the wire).

High-quality quantum wires can be fabricated by the molecular-beam epitaxy using the cleaved edge overgrowth (CEO) technique [3], as proposed by Esaki et al. [4. Such structures are formed at the T-shaped intersection of two semiconductor quantum wells, see fig. 1. The electronic band structure of realistic T-shaped AlGaAs/GaAs quantum wires has been studied numerically using different methods [5.

Note, however, that the form of the T-shaped confinement potential, fig. 1, does not allow for analytical solutions of the one-electron continuum Schrödinger equation even in the limiting case of infinite energy barriers and isotropic electron effective mass. Meanwhile, the existence of electron states localized in the region of intersection of two quantum wells within the plane perpendicular to the axis of CEO quantum wire is not obvious a priori. In this paper, we propose a simple model of the two-dimensional T-shaped structure and obtain an analytical solution for both the lowest energy eigenvalue and corresponding localized eigenfunction of the one-electron Schrödinger equation.

Let us consider two intersecting atomic chains, one of which (along $x$-axis) is infinite, while another (along $y$-axis) is semi-infinite, see fig. 2. Such a system provides a model for the description of electron motion in the $x y$-plane perpendicular 
to the axis of a CEO quantum wire (fig. 1) since an electron is confined to the chains and can not escape into the interjacent region. This model corresponds to the limiting case of a CEO quantum wire formed by two quantum wells with one-atomic-layer width and an infinite value of the energy barrier $V=E_{c}^{A}-E_{c}^{B}$ preventing an electron from going to the conduction band of the semiconductor $\mathrm{A}$, see fig. 1.

In a tight-binding approach, one-electron states of the system under consideration are described by the following Hamiltonian:

$$
\hat{H}(t)=\epsilon_{0} \sum_{i} \hat{a}_{i}^{+} \hat{a}_{i}-t \sum_{<i, j>}\left(\hat{a}_{i}^{+} \hat{a}_{j}+h . c\right)
$$

where $\epsilon_{0}$ is an on-site potential, $t$ is a matrix element for electron hopping between nearest sites $i$ and $j, \hat{a}_{i}^{+}\left(\hat{a}_{i}\right)$ is the operator of electron creation (annihilation) at the site $i$, and $\langle i, j\rangle$ means the summation over nearest neighbors only. We do not specify the spin index explicitly since we consider the states of a single electron.

Expanding the one-electron wave function $\Psi$ into atomic electron states $\mid i>$,

$$
\Psi=\sum_{i} A_{i} \mid i>
$$

we have from the Schrödinger equation $\hat{H} \Psi=E \Psi$ a set of algebraic equations for coefficients $A_{i}$ whose squared absolute values $\left|A_{i}\right|^{2}$ give the probabilities to find an electron at a particular site $i$ :

$$
\tilde{E} A_{i}=\sum_{j=n n(i)} A_{j}
$$

where

$$
\tilde{E}=\frac{\epsilon_{0}-E}{t}
$$

and $j=n n(i)$ means the summation over sites $j$ nearest to the site $i$.

Let $i=n$ for atoms in the chain running along $x$-axis and $i=m$ for atoms in the chain along $y$-axis $(n=0, \pm 1, \pm 2, \pm 3, \ldots ; m=0,1,2,3, \ldots)$, see fig. 2 . For the 
atom belonging to both chains (at the intersection point) one has $n=m=0$. We consider three different regions: (1) $n \geq 0$; (2) $n \leq 0$; (3) $m \geq 0$. In those regions, we have from eq. (3):

$$
\begin{gathered}
\tilde{E} A_{n}=A_{n-1}+A_{n+1}, n \geq 1, \\
\tilde{E} A_{n}=A_{n-1}+A_{n+1}, n \leq-1, \\
\tilde{E} A_{m}=A_{m-1}+A_{m+1}, m \geq 1 .
\end{gathered}
$$

General solutions of eqs. (5) are:

$$
\begin{gathered}
A_{n}=A_{0} \exp (-\alpha n), n \geq 0, \\
A_{n}=B_{0} \exp (\beta n), n \leq 0, \\
A_{m}=C_{0} \exp (-\gamma n), m \geq 0,
\end{gathered}
$$

where

$$
\tilde{E}=2 \cosh (\alpha)=2 \cosh (\beta)=2 \cosh (\gamma)
$$

Since, due to normalization condition $\sum_{i}\left|A_{i}\right|^{2}=1$, the coefficients $A_{n}$ and $A_{m}$ should be finite at $n \rightarrow \pm \infty$ and $m \rightarrow \infty$ respectively, one has $\operatorname{Re}(\alpha) \geq 0, \operatorname{Re}(\beta) \geq$ 0, $\operatorname{Re}(\gamma) \geq 0$. Hence, we see from eq. (10) that $\alpha=\beta=\gamma$, while from eqs. (6) we have $A_{0}=B_{0}=C_{0}$ since $A_{n=0}=A_{m=0}$ at the intersection point.

Next, from eq. (3) for the coefficient $A_{0}$ we obtain

$$
\tilde{E} A_{0}=A_{n=1}+A_{n=-1}+A_{m=1} .
$$

Taking eqs. (6) into account, we have

$$
\tilde{E}=3 \exp (-\alpha)
$$

The lowest energy solution of eqs. (7) and (9) is $\tilde{E}=3 / \sqrt{2}$ and $\exp (-\alpha)=1 / \sqrt{2}$, and so from eq. (雨) we have the minimum one-electron energy of the system under 
consideration:

$$
E_{\text {min }}=\epsilon_{0}-\frac{3}{\sqrt{2}} t
$$

while the corresponding normalized wave function is

$$
\Psi=\sum_{n=-\infty}^{\infty} \frac{1}{2^{|n| / 2+1}}\left|n>+\sum_{m=1}^{\infty} \frac{1}{2^{m / 2+1}}\right| m>.
$$

One can see from eq. (11) that the wave function is localized exponentially in the vicinity of the intersection point $n=0, m=0$. The probability to find an electron at the site $n=m=0$ equals to $1 / 4$, while the probabilities to find an electron at the nearest sites $n=-1, n=1, m=1$ are $1 / 8$ each. The localization length $\xi$ defined by $A_{n}=\exp (-a|n| / \xi)$, where $a$ is the interatomic spacing, equals to $\xi=a / \alpha=2 a / \ln (2) \approx 3 a$.

We note that the value of $E_{\text {min }}=\epsilon_{0}-3 t / \sqrt{2}$ is by $\approx 0.12 t$ lower than the edge $E_{0}=\epsilon_{0}-2 t$ of the band of delocalized electron states in the infinite one-dimensional chain. An estimate of the hopping matrix element $t$ for a specific case of GaAs gives in a tight-binding approach $t=\hbar^{2} / 2 m^{*} a^{2} \approx 1.8 \mathrm{eV}$, where $m^{*}=0.067 m_{0}, a=$ $0.565 \mathrm{~nm}, m_{0}$ is the mass of a free electron. Then for the confinement energy one has $E_{c}=E_{0}-E_{\text {min }} \approx 200 \mathrm{meV}$. The physical reason for appearance of the localized state in the T-shaped geometry seems to be related with the effect of Anderson localization [6] since the semi-infinite chain along $y$-axis plays a role of defect for an electron moving in the infinite chain along $x$-axis.

We have also studied the model under consideration by the exact numerical diagonalization of the Hamiltonian matrix. We made use of the complex conjugate method which allows one to find eigenvalues and eigenvectors of large sparse matrixes with any prescribed accuracy [7]. For our purposes, we have restricted ourselves to several low-lying levels. We have considered the systems composed of $3 N+1$ sites $(2 N+1$ sites in the chain along $x$-axis and $N+1$ sites in the chain along $y$-axis, one 
site being common for both chains), with $N$ up to $10^{3}$. For the ground state, the calculated values of $E_{\min }$ and $\alpha$ coincide with analytical results. The excited states have the energies $E_{i} \geq E_{0}$, where the value of $E_{0}$ tends to $\epsilon_{0}-2 t$ as $N$ increases, e.g., $E_{0}=\epsilon_{0}-1.99903 t$ for $N=100$ and $E_{0}=\epsilon_{0}-1.99976 t$ for $N=200$. The mean level spacing of the excited states is of the order of $t / N$ and goes to zero as $N$ increases. An inspection of wave functions of low-lying excited states has shown that all of them are delocalized over the whole system, having the form of sines or cosines.

Finally, along with the system shown in fig. 2, we have examined a more general case of quasi-one-dimensional chains of finite width, so that each chain had $N_{0} \geq 2$ sites in width $\left(N_{0}=1\right.$ in a particular case studied analytically above). We have considered the systems composed of $3 N \cdot N_{0}+N_{0}^{2}$ sites $\left(\left(2 N+N_{0}\right) \cdot N_{0}\right.$ sites in the chain along $x$-axis and $\left(N+N_{0}\right) \cdot N_{0}$ sites in the chain along $y$-axis, $N_{0}^{2}$ sites being common for both chains), with $N$ up to $10^{4}$ and $N_{0}$ up to 40 . We have found that the ground state energy $E_{\text {min }}$ decreases with increasing $N_{0}$, see fig. 3 , and remains lower than the edge of the band of delocalized states, $E_{0}$. Fig. 4 shows the confinement energy $E_{c}=E_{0}-E_{\min }$ as a function of the chain width $N_{0}$. One can see that $E_{c}$ decreases with $N_{0}$ and tends to zero as $N_{0}$ goes to infinity. Taking the values of $t$ and $a$ for GaAs, see above, one has, e.g., $E_{c} \approx 32 \mathrm{meV}$ and $9 \mathrm{meV}$ for the chains of $5 \mathrm{~nm}$ and $10 \mathrm{~nm}$ width respectively. These values of $E_{c}$ are about twice as large as those obtained for the conduction band of symmetrical T-shape wire structures with GaAs wells and $\mathrm{Al}_{0.3} \mathrm{Ga}_{0.7}$ As barriers within a much more sophisticated model [5]. An apparent reason for this quantitative discrepancy is the infinite value of the conduction-band offset in our simple model. So, our approach provides an upper estimate for the electron confinement energy in the T-shaped quantum wires. 
From figs. 3 and 4 we find that at large $N_{0}$ both $E_{\min }$ and $E_{0}$ approach $-4 t$ which is just the value of $E_{0}=E_{\min }$ for the infinite two-dimensional system in a tight-binding model. Again, the ground state wave function is always localized exponentially in the vicinity of the intersection region, while the wave functions of excited states are delocalized. The values of the localization length $\xi$ at different $N_{0}$ where found through the analysis of the asymptotic behavior of the ground state wave function at large distance from the intersection region. It was found that the decrease in $E_{c}$ with $N_{0}$ is accompanied by the corresponding increase in $\xi$ according to the general relation $E_{c}=\hbar^{2} / 2 m^{*} \xi^{2}=t(a / \xi)^{2}$. The dependence of $\xi$ on $N_{0}$ is shown in fig. 5 .

In conclusion, we have presented an analytically solvable model of the T-shaped two-dimensional confinement potential. Although being rather simple, this textbook model explicitly demonstrates the existence of localized electron states in the Tshaped geometry. It can be used for qualitative estimates of confinement energies and the extent of spatial localization of one-electron wave function in CEO quantum wires. The model can be easily generalized to account for electron hopping to atoms other than nearest neighbors only and/or for the finite value of the confinement potential. In such a case, however, it will be difficult to obtain an analytical solution of one-electron Schrödinger equation.

This work was supported in part by the Russian Federal Program "Integration", project No A0133. 


\section{References}

[1] SCHULZ H. J., in Mesoscopic Quantum Physics, edited by E. AKKERMANS, G. MONTAMBEAUX, J.-L. PICHARD and J. ZINN-JUSTIN (Elsevier, Amsterdam) 1995.

[2] BeEnAKkeR C. W. J. and VON HOUTEN H., in Solid State Physics, Semiconductor Heterostructures and Nanostructures, edited by H. EHRENREICH and D. TURNBULL (Academic Press, New York) 1991.

[3] PFEIFFER L., WEST K. W., STÖRMER H. L., EISENSTEIN J. P., BALDWIN K. W., GerShOni D. and SPECTOR J., Appl. Phys. Lett., 56 (1990) 1697.

[4] CHANG Y.-C., CHANG L. L. and ESAKI L., Appl. Phys. Lett., 47 (1985) 1324.

[5] For example, LANGBEin W., GISLASON H. and HVAM J. M., Phys. Rev. B, 54 (1996) 14595; GOLDONI G., ROSSI F., MOLINARI E. and FASOLINO A., Phys. Rev. B, 55 (1997) 7110.

[6] ANDERSON P. W., Phys. Rev., 109 (1958) 1492.

[7] OPENOV L. A., Phys. Low-Dim. Struct., 10/11 (1995) 365. 


\section{FIGURE CAPTIONS}

Fig. 1. Schematic view of a T-shaped semiconductor quantum wire formed by two intersecting quantum wells. The height of the energy barrier for electrons in

the semiconductor B equals to the conduction-band offset $\Delta E_{c}=E_{c}^{A}-E_{c}^{B}$ between semiconductors A and B. Electrons are confined in the $x y$-plane in the vicinity of intersection of quantum wells and move freely along $z$-axis (an axis of the quantum wire)

Fig. 2. Two intersecting atomic chains as a model of a T-shaped confinement potential for electrons in the plane perpendicular to the axis of CEO quantum wire. Numbers $n$ and $m$ numerate atoms in the chains along $x$ - and $y$-axis respectively.

Fig. 3. The energy $E_{\min }$ of the localized state in units of the hopping matrix element $t$ versus the number of sites $N_{0}$ across the chain. Points are the results of analytical (for $N_{0}=1$ ) and numerical (for $N_{0} \geq 2, N=800$ ) calculations. The line is a guide to the eye.

Fig. 4. The confinement energy $E_{c}=E_{0}-E_{\min }$ in units of the hopping matrix element $t$ versus the number of sites $N_{0}$ across the chain. Points are the results of analytical (for $N_{0}=1$ ) and numerical (for $N_{0} \geq 2, N=800$ ) calculations. The line is a guide to the eye.

Fig. 5. Localization length $\xi$ of the ground state wave function in units of the interatomic spacing $a$ versus the number of sites $N_{0}$ across the chain. Points are the results of analytical (for $N_{0}=1$ ) and numerical (for $N_{0} \geq 2, N=800$ ) calculations. The line is the curve $\xi=a\left(t / E_{c}\right)^{1 / 2}$, where $E_{c}=E_{0}-E_{\min }$ is the confinement energy. 
A

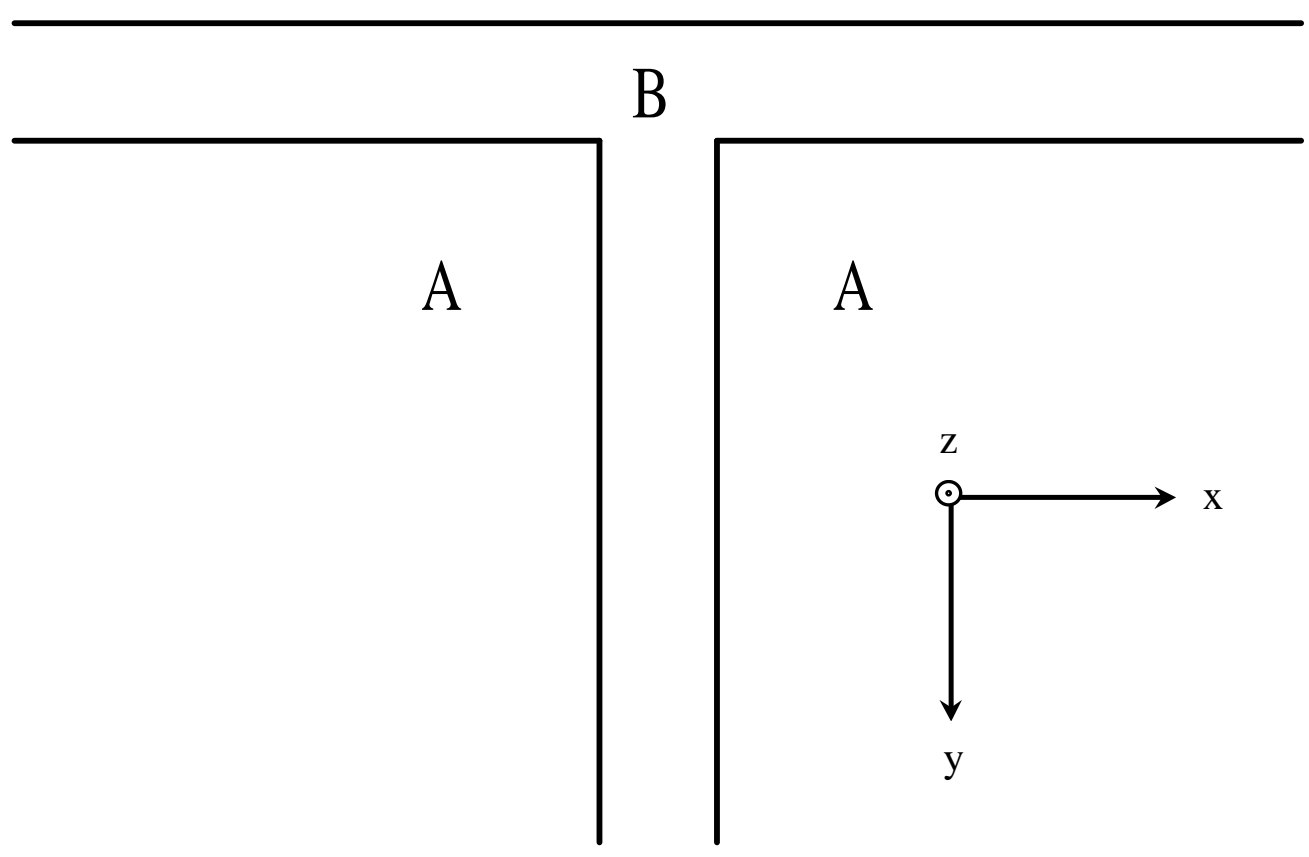




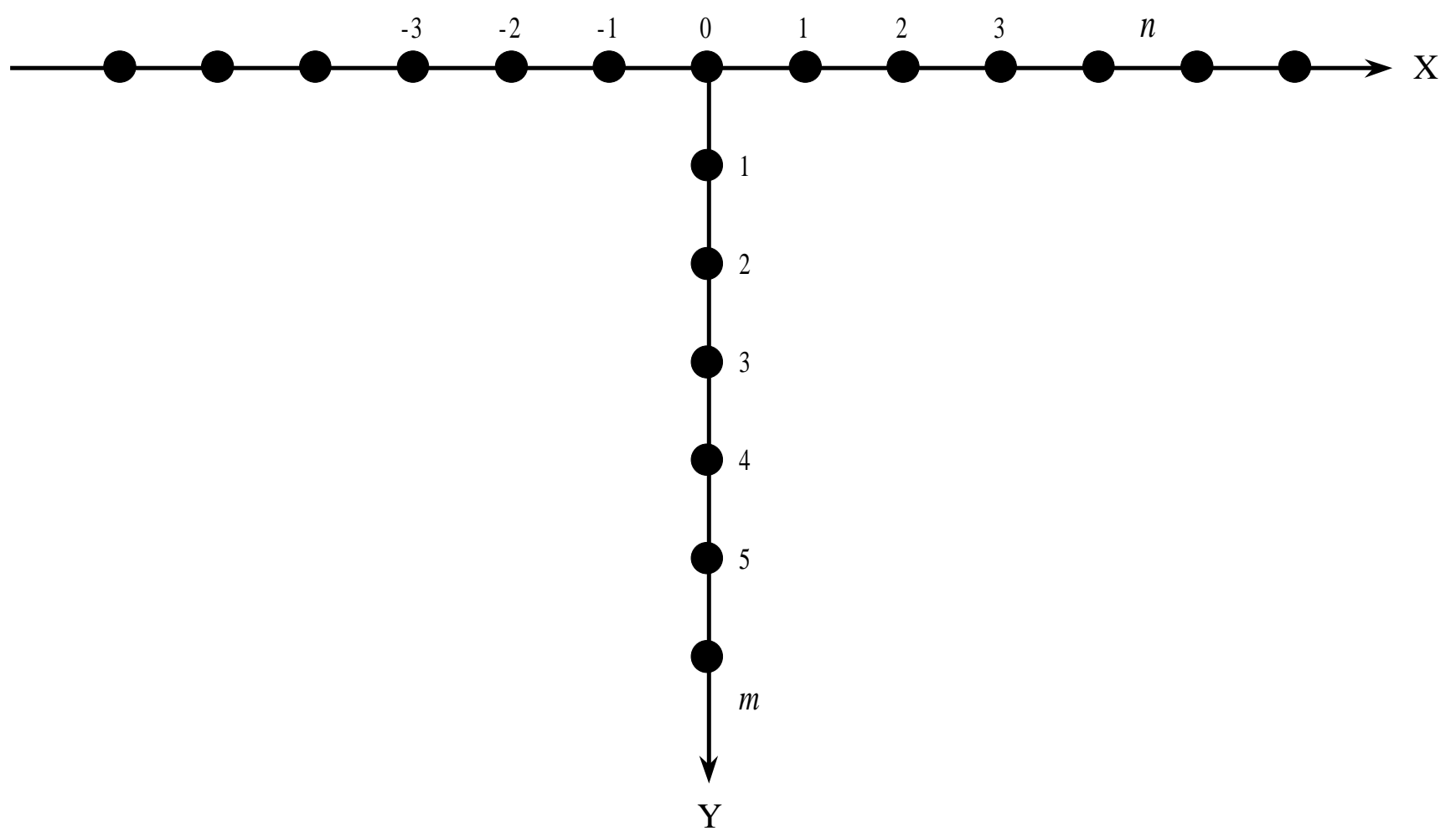




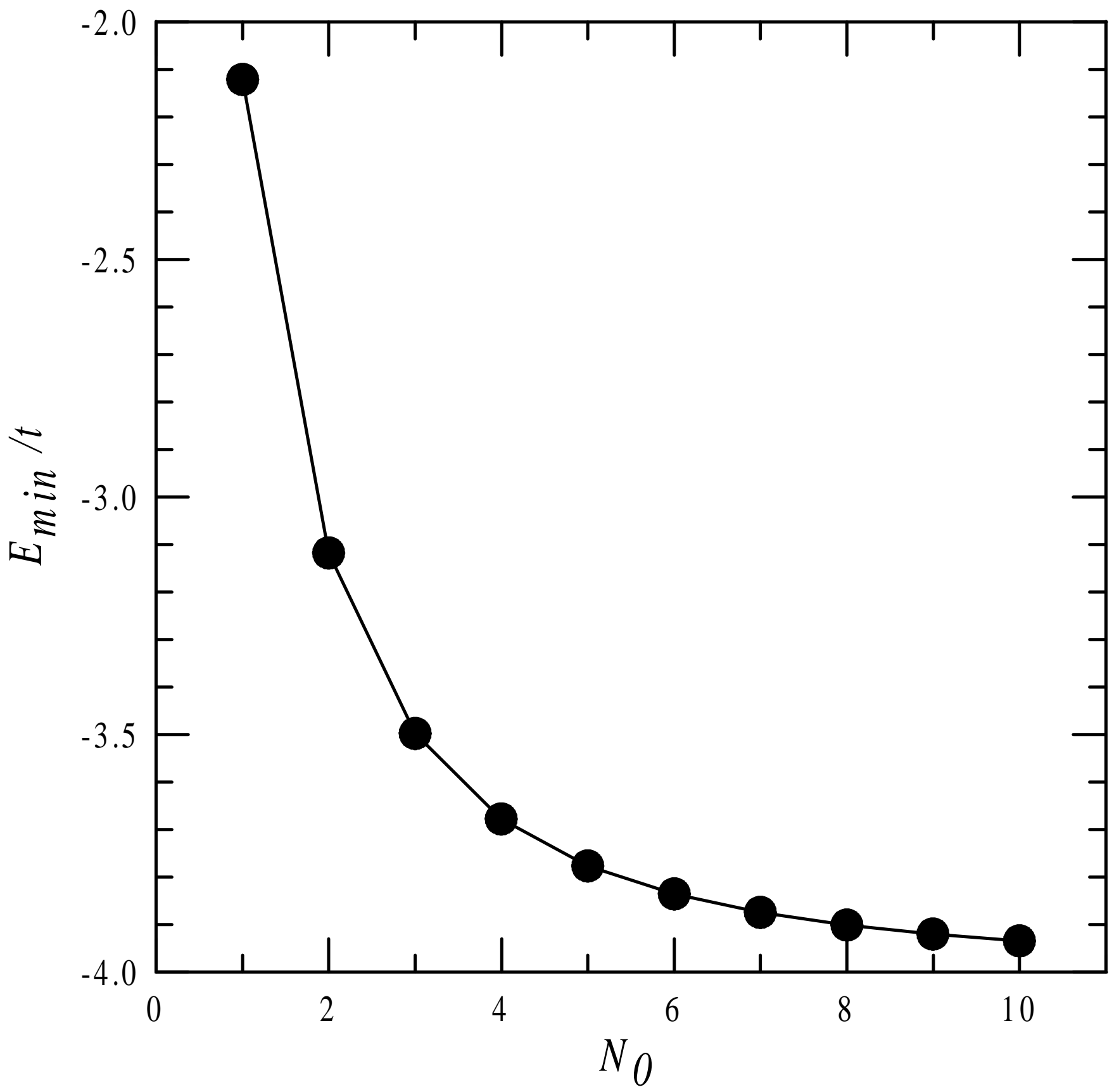




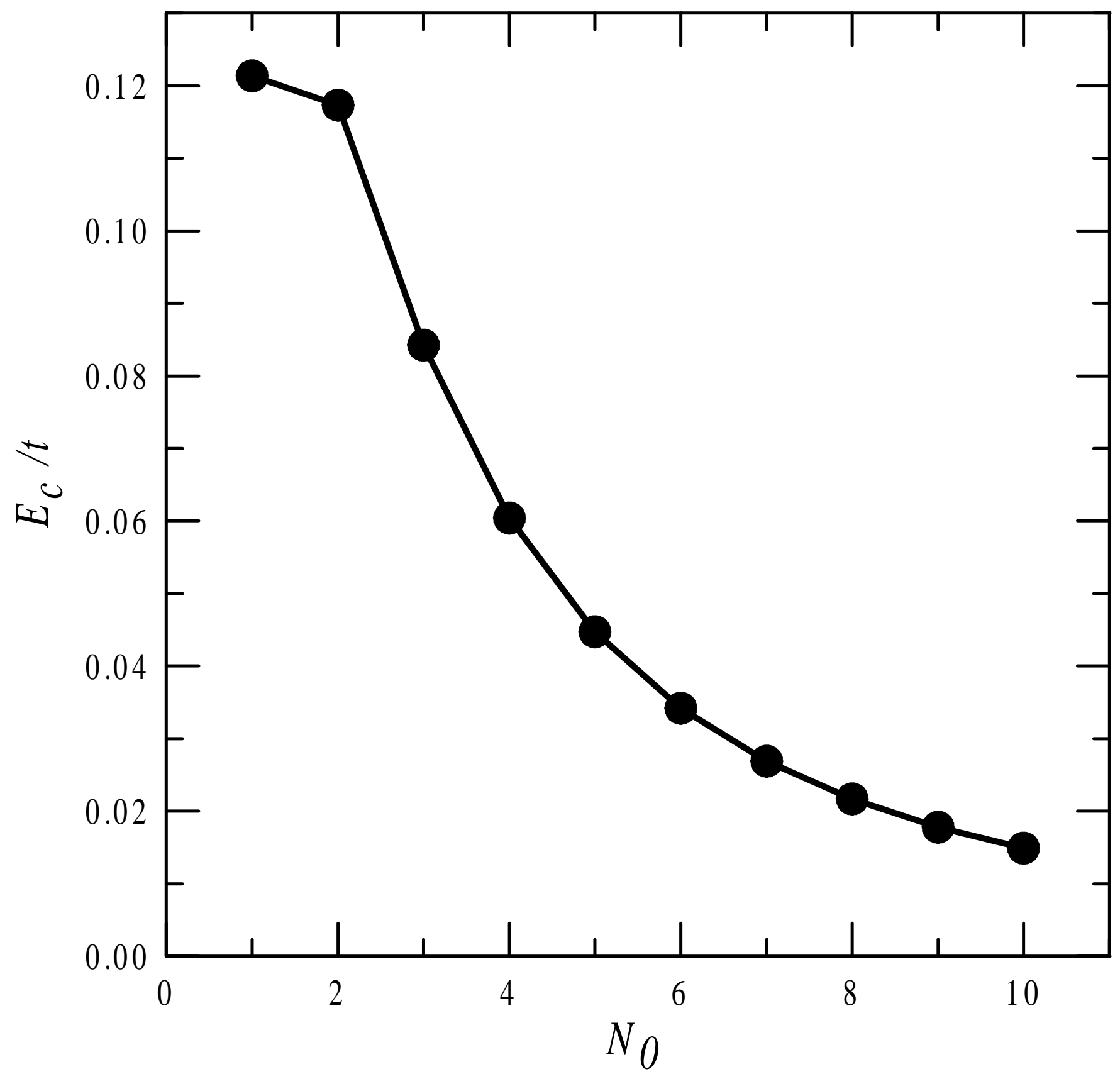




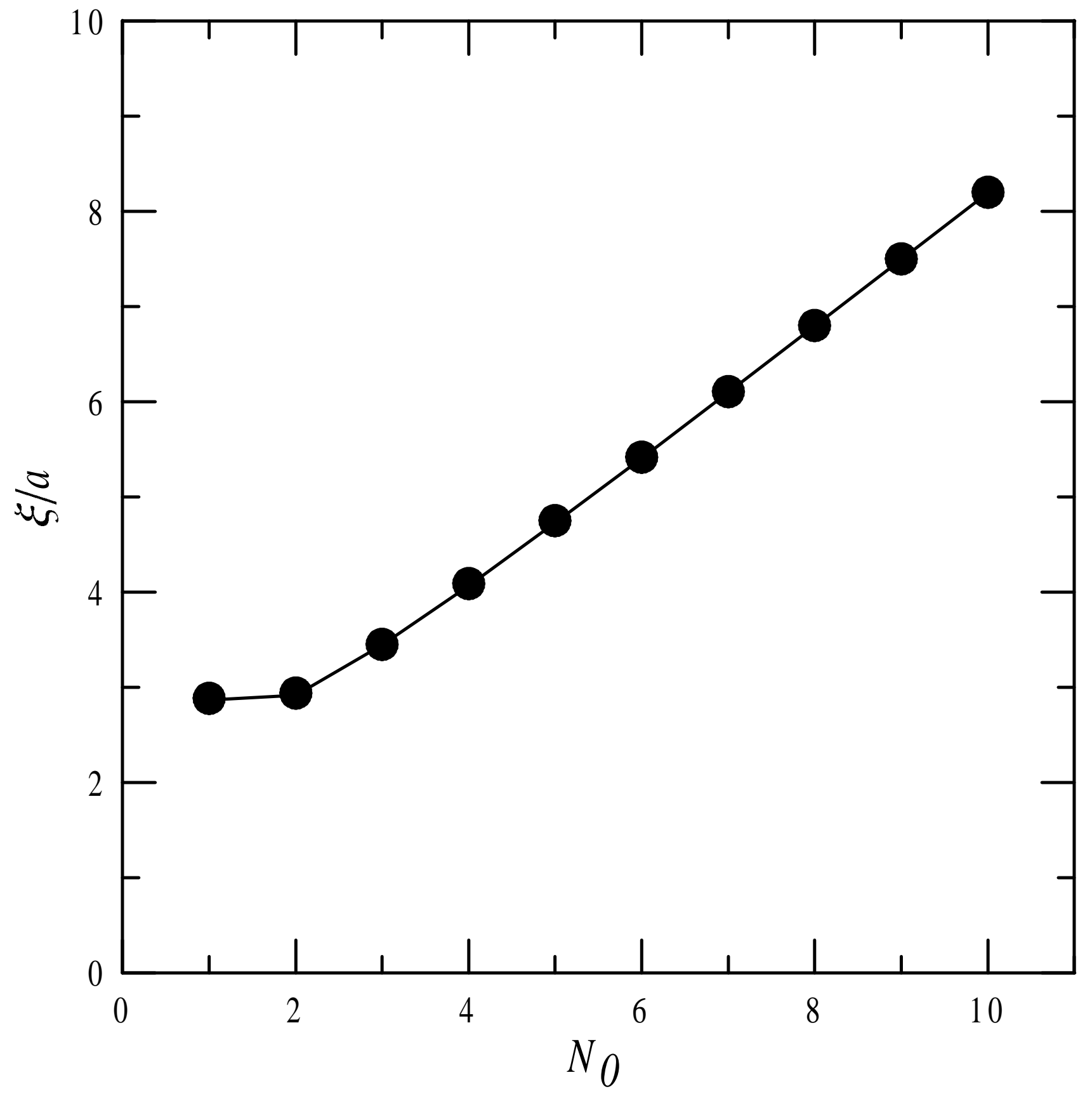

